\title{
Multi-Temporal Analysis of Forestry and Coastal Environments Using UASs
}

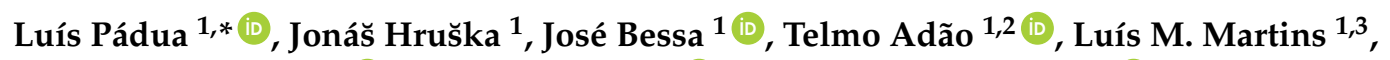 \\ José A. Gonçalves $^{4,5}$ (D), Emanuel Peres ${ }^{1,2}$ (D) , António M. R. Sousa ${ }^{1,2}$ (D) , João P. Castro 6 \\ and Joaquim J. Sousa 1,2,* (iD) \\ 1 Department of Engineering, School of Sciences and Technology, University of Trás-os-Montes e Alto Douro, \\ 5000-801 Vila Real, Portugal; jonash@utad.pt (J.H.); jmiguelbessa16@gmail.com (J.B.); \\ telmoadao@utad.pt (T.A.); lmartins@utad.pt (L.M.M.); eperes@utad.pt (E.P.); amrs@utad.pt (A.M.R.S.) \\ 2 INESC-TEC (formerly INESC Porto), 4200-465 Porto, Portugal \\ 3 CITAB - Centre for the Research and Technology of Agro-Environmental and Biological Sciences, \\ 5000-801 Vila Real, Portugal \\ 4 Department of Geosciences, Environment and Land Planning, Science Faculty, University of Porto, \\ 4169-007 Porto, Portugal; jagoncal@fc.up.pt \\ 5 CIIMAR-Interdisciplinary Centre of Marine and Environmental, 4450-208 Matosinhos, Porto, Portugal \\ 6 CIMO-Mountain Research Centre, ESAB, Polytechnic Institute of Bragança, 5300-253 Bragança, Portugal; \\ jpmc@ipb.pt \\ * $\quad$ Correspondence: luispadua@utad.pt (L.P.); jjsousa@utad.pt (J.J.S.); Tel.: +351-259-350-356 (L.P. \& J.J.S.)
}

Received: 6 November 2017; Accepted: 19 December 2017; Published: 24 December 2017

\begin{abstract}
Due to strong improvements and developments achieved in the last decade, it is clear that applied research using remote sensing technology such as unmanned aerial vehicles (UAVs) can provide a flexible, efficient, non-destructive, and non-invasive means of acquiring geoscientific data, especially aerial imagery. Simultaneously, there has been an exponential increase in the development of sensors and instruments that can be installed in UAV platforms. By combining the aforementioned factors, unmanned aerial system (UAS) setups composed of UAVs, sensors, and ground control stations, have been increasingly used for remote sensing applications, with growing potential and abilities. This paper's overall goal is to identify advantages and challenges related to the use of UAVs for aerial imagery acquisition in forestry and coastal environments for preservation/prevention contexts. Moreover, the importance of monitoring these environments over time will be demonstrated. To achieve these goals, two case studies using UASs were conducted. The first focuses on phytosanitary problem detection and monitoring of chestnut tree health (Padrela region, Valpaços, Portugal). The acquired high-resolution imagery allowed for the identification of tree canopy cover decline by means of multi-temporal analysis. The second case study enabled the rigorous and non-evasive registry process of topographic changes that occurred in the sandspit of Cabedelo (Douro estuary, Porto, Portugal) in different time periods. The obtained results allow us to conclude that the UAS constitutes a low-cost, rigorous, and fairly autonomous form of remote sensing technology, capable of covering large geographical areas and acquiring high precision data to aid decision support systems in forestry preservation and coastal monitoring applications. Its swift evolution makes it a potential big player in remote sensing technologies today and in the near future.
\end{abstract}

Keywords: multi-temporal data analysis; unmanned aerial systems; chestnut trees; coastal environments

\section{Introduction}

Unmanned aerial systems (UASs) allow professionals, acting in different areas of society, to capture up-to-date, high resolution, and accurate positional data that may be used for generating 
advanced data products—such as 3D point clouds, orthomosaics (orthophotomaps), digital surface models (DSMs) and vegetation indices-which are very useful for classification and segmentation. As occurs in many other technological fields, the number of applications is increasing every day, causing a rapid increase in UAS usage around the world, with exponential growth expected in the coming years [1,2].

The main factors supporting this growth are related to the: (1) increasing awareness about the benefits that this kind of technology can bring to a wide range of industries and non-commercial sectors; (2) introduction of relatively low-cost systems, and user-friendly controls, as well as general technological advancements and miniaturization of individual components and; (3) the introduction of pragmatic and business-friendly UAS legislation.

According to the Association of Unmanned Vehicle System International (AUVSI), the integration of UASs into the United States national airspace in the decade 2015-2025 is expected to create more than 100,000 jobs and generate an economic impact of $\$ 82$ billion [3].

As a new method of geo-data collection, UASs complement existing techniques, filling the gap between large area imaging (satellites and manned aircrafts) and smaller coverage, time-consuming, but highly accurate terrestrial techniques [4]. Compared to high altitude data, UAS data is fairly low cost, with the advantage of allowing frequent and flexible flights [5]. UASs are thus very useful when small and medium-sized land parcels need to be frequently surveyed, allowing a rapid response option for time-sensitive deliverables, disaster situations, or search and rescue operations.

Moreover, the use of UAS brings the benefit of performing inventory analysis based on the collection and archive of aerial imagery, allowing temporal comparison. Recently, multi-temporal analysis has been explored by several authors [6-8]. In contrast, traditional aerial methods of data acquisition (e.g., satellites and manned aircrafts) may be limited for this type of analysis, due to the high cost involved in obtaining repeated imagery [9].

Given their very specific characteristics, UASs have been progressively used in several research applications, covering a growing diversity of fields that range from public safety [10] and infrastructures inspections [11], to environmental conservation/preservation [12-14], agriculture [15], and forestry [16]. It is precisely in the latter for which the greatest economic impacts are expected to occur in the next decade [3]. A detailed review of the different types of UASs and applications can be found in the work developed by Colomina and Molina [17].

Considering the mentioned applications there are two fields of particular interest: (1) forestry preservation/conservation; and (2) coastal monitoring for prevention purposes. Regarding the former, diseases and pests cause tremendous economic losses and drastically reduce the quality of many cultivated crops, as well as wild vegetation species, which is of economic relevance for respective exploring communities. Therefore, early detection and assessment of crop symptoms and damage are crucial for plant health [18]. When in presence of biotic stress, the disease's damage mechanism influences the plants' physiological response, which manifests through certain symptoms (e.g., wilting, stunted growth, reduction in leaf/canopy area, chlorosis or necrosis in some parts, leaf curling), creating some difficulties when trying to obtain an accurate quantification of the affected plants, usually by direct observation in the field. Remote sensing platforms such as the UAS can provide an alternative and cost-effective method, allowing the application of non-destructive and non-invasive methods to obtain accurate spatial data for entire crop fields at frequent intervals [19]. In the same way, this technology can aid in the prediction and prevention of occurrences related to coastal environments wherein the water progress-for example, caused by coastal perimeter degradation [14], may cause serious problems for local businesses and dwellers as well.

In this paper, two case studies based on the analysis of multi-temporal data acquired using UASs are presented. The first study concerns the monitoring of the health status of chestnut trees in Portugal, particularly in the Padrela region (the north-eastern part of the country). This region generates the highest production of chestnuts in the country [20], representing the greatest source of income for the entire region. The second case study is associated to one of the main challenges for the next 
years: the identification of ways to reduce/reverse the effects of climate change, which are causing alterations to natural resources and ecosystem dynamics. The coastal zones are some of the most affected areas, making sand dune protection an important issue due to their role in coastal defense. In fact, sand dunes play a crucial role in fauna and flora protection and provide sediment supplies to maintain the beaches that, in turn, are responsible for the protection of coastal agriculture systems and inland areas from storms and the rise in sea level [21,22]. Therefore, the second study presented in this paper focuses on the application of UAS multi-temporal data for monitoring the erosion occurring in coastal zones, particularly in the Cabedelo area where one of the most important and sensitive natural areas of Portugal is located: the Cabedelo sandspit, located in the Douro River estuary (Porto, Portugal). This natural structure is responsible for the preservation of ecosystems and for the protection of the sand area. Thus, these dunes are crucial for preserving the dwellings situated on the coastline as well as those of the local population. In each one of the referred case studies, flights were carried out at different times under similar conditions-light, temperature, etc. to ensure radiometric and geometric consistency.

This paper's main objectives are: (1) to identify the advantages and challenges associated with the use of a reliable, robust and cost-effective solution-using UAS to acquire aerial imagery data in forestry and coastal monitoring contexts; and (2) to demonstrate this remote sensing platform flexibility to cover such distinct environments. Moreover, the benefits of multi-temporal analysis in change detection will also be explored. Five sections comprise this paper: after this introduction, Section 2 presents a background on UASs including historical context, supported sensors, achievable products, and several applications towards environmental monitoring. In Section 3, the investigation methodology addressing data collection and processing is presented. Case studies are described in Section 4. The paper finishes with conclusions and future perspectives in Section 5.

\section{Background}

In the last 60 years, with developments in electronics, computing, and remote sensing, technological has advanced and platforms suitable for aerial data acquisition have been produced. With respect to this topic, satellites have been the most used system over the past 30 years [23]. However, its use can represent a high cost when studying small or medium-sized areas [5], which occurs in many remote sensing applications in the scope of forestry and coastal environments. Ponti [24] suggests the adoption of an alternative technology, such as UASs. This technology presents itself as a viable alternative to satellites, mostly because of [25]: (1) the higher temporal (up to daily) data acquisition and the higher spatial (up to millimetric) resolution of acquired imagery it typically offers $[16,26]$; (2) its ease in terms of scheduling and programming image acquisition operations; (3) its flexibility to operate in different environments (often with difficult access); and (4) its versatility, since surveys can be conducted in different contexts and extensions/heights.

Sensors coupled to unmanned aerial vehicles (UAVs) represent the most important system part, as it is through them that data will be acquired and, therefore, valuable data products will be generated(e.g., orthomosaics, DSMs, 3D point clouds, vegetation indices) [27-30]. Sensors are classified as active or passive and a large variety can be found. Regarding passive sensors, they are used for detecting natural emissions from both the atmosphere and the Earth's surface (e.g., red-green-blue (RGB), near-infrared (NIR), and thermal emissions), while active sensors transmit their own radiation pulses through their energy sources (e.g., light detection and ranging (LIDAR), radio detection and ranging (RADAR)) [4].

The interest in the UAS as a form of remote sensing technology has grown because it allows user-controlled image acquisition and fills the gap — both in scale and resolution-between terrestrial observations and conventional manned aircrafts and satellite sensors. It is a cost-effective solution and enables adapting acquired imagery of the observed objects' real dimensions to the monitored processes and to alteration speed within a given landscape [31]. When compared with traditional remote sensing 
platforms for imagery acquisition, UASs are considered both more effective and accurate when used in areas up to $10 \mathrm{~km}^{2}$ [32].

Despite the many advantages of remote sensing technologies, it is necessary to consider that there are some factors that may limit their performance, such as [33]: frame mosaicking, band-to-band registration, natural dynamics (such as atmospheric conditions), the Sun's angle, and technical problems (like viewing angle definition or changes in sensor calibration over time). However, if the best usage/operation practices are ensured at the pre-processing stage and during image acquisition, such limitations can be mitigated. In what concerns geographic data acquisition, UAS application is of superlative importance when addressing areas where access is difficult or dangerous by conventional means. UASs can be used for several purposes, such as [34,35]: spotting, tracking, and fighting fires; support in natural disaster scenarios, timely distribution of medication and aid; air quality monitoring; wildlife surveys (e.g., survey migration flows); crime fighting, surveillance tasks and other protection-related activities; delivering products; monitoring natural phenomena so that preventive actions can be taken; 3D mapping; and search and rescue actions. However, it is worth noting that when measuring and mapping activities are performed, it is imperative to geocode and geometrically correct the acquired images [36].

The number of scientifically published case studies involving UAVs as remote sensing platforms is growing, making the application of UASs an interesting subject, especially in the environmental field for tasks such as: weed control monitoring [37]; crop pest management [38]; Artic sea ice and atmosphere monitoring [39]; soil properties monitoring [2]; vineyard vigor mapping [40]; water monitoring [41]; habitat mapping [42]; and landslide dynamics [43]. The diversity of these contributions clearly shows the increasing importance of UASs for remote environmental monitoring.

In addition to these scientific contributions, those which involve protection and preservation of ecosystems' dynamics in coastal zones and vegetation monitoring are considered especially relevant to this paper. During the last years, coastal zones have suffered significant erosion, primarily due to the rising sea action, wind, and storms, mostly triggered by climate change. As such, beaches have experienced rapid morphological changes, which means that it is even more important to preserve natural barriers such as sand dunes [44]. Topographical changes in beaches and natural barriers need to be monitored and assessed on a regular basis to build models and to simulate scenarios that can help in predicting these natural environments evolution. Nowadays, UASs represent a valuable tool to provide data to compute scenarios and monitor events [45]. For instance, UASs were applied for quantifying the coastal impact before and after a storm, allowing for the monitoring of the evolution of a rubble-mound breakwater on the mid-New South Wales (NSW) Australian coast and mapping of the vegetation in a coastal estuary entrance [46]. Messinger and Silman [47] investigated the suitability and application of UASs in environmental emergency response, in the case of coal ash spills. In Portugal, this kind of technology was used for surveillance and control of maritime traffic, fishing surveillance, and the detection/control of coastal hazards [48]. Hodgson et al. [49] investigated the conservation and management of marine fauna through the application of UAS, to monitor mammal species population status. Rhee et al. [50] applied this remote sensing technology on fluvial waters, with the objective of monitoring riparian vegetation, hazardous aquatic algae blooms and submerged morphology, and water-surface slope, among other phenomena.

Regarding vegetation monitoring, protecting and increasing food and water supplies for a global population that is growing quickly and in an exponential manner must be a priority [51]. Indeed, crop management becomes a critical factor to maximize yield while reducing and environmental risks and impacts where UAS platforms have been playing an important role in this context. Some of applications include tree canopy health mapping in a macadamia orchard for plantation management purposes [52], early site-specific weed detection in wheat fields [53], automated crop lodging detection in maize [54], and vegetation filtering for river riparian zones [55].

UAS flexibility increases its applicability for surveying of the same area over time, especially in very dynamic environments requiring close monitoring, which is not possible-at least, in a 
cost-effective manner-by means of other remote sensing platforms. This approach has already been applied in some studies, where this remote sensing technology has been used to acquire multi-temporal data with different purposes, in several types of agricultural crops, such as barley [56], sunflowers [57], silage maize [58], rice [59], wheat [60,61], and vineyards [62]. In the aforementioned studies, multi-temporal imagery acquisition gave results that, in some cases, were noticeable only after a certain vegetative cycle stage of the studied crops. Moreover, this approach was already employed in coastal environments [63], assessment of landslides displacements [64], and in monitoring of forest growth and biomass estimation [65].

Thus, applying UASs can be advantageous for monitoring certain areas, since it allows to assess them and to identify potential problematic zones, and/or to evaluate implemented mitigation/prevention measures in an effective way. The ext section will address the methodology used in the two case studies presented in Section 4, which benefit from the usage of multi-temporal UAS-based imagery.

\section{Methodology}

This section describes the methodology used in the presented case studies. The applied methodology was similar for both studies and consists essentially of two phases: (1) field work-data collection by acquiring high-resolution images using UASs and, when necessary, some ground control points (GCPs); and (2) data processing-manipulation and analysis of the collected data (through specific software) to produce valuable and meaningful information. Since atmospheric influence is of minor impact while using UASs for land surveying [4] and also because conditions-e.g., light and temperature-were consistently ensured between flights, radiometric corrections were considered negligible for both addressed case studies (monitoring of Padrela's chestnut trees and Cabedelo's sandspit) and, thus, they were not performed. These stages are further described in the following subsections.

\subsection{Data Collection}

The selection of a UAV for data acquisition is influenced by the specificities of each case study. In that selection, some characteristics have to be considered: ground sample distance (GSD); minimum coverage; ability to be deployed in rugged terrain, ability to operate from unprepared surfaces and in constrained conditions; autonomy of at least $30 \mathrm{~min}$; being easy to carry over long distances; ease of simple field maintenance and reparability; reduced environmental emissions and noise signature; and reliable and low cost. The UAVs used in the case studies presented in this paper are described in Section 4. All flights were conducted in parallel rows with the minimum longitudinal overlap of $75 \%$ and lateral overlap of $60 \%$ [63]. The flights were planned by using specific software, wherein the user defines the area of interest, flight direction, longitudinal and lateral overlapping, and pixel-size on the ground (GSD) (Figure 1).

The imagery used in this study was collected using the Canon IXUS 127 HS camera (16 mega-pixels) and the Canon PowerShot ELPH 110 HS camera (12 mega-pixels). The former provided the possibility of collecting images in the visible part of the electromagnetic spectrum, i.e., red-green-blue (RGB) bands, while the latter allowed the collection of imagery in RedEdge (RE), green and blue bands. RE is the spectral region where the plant's reflectance changes from low to high (from $680 \mathrm{~nm}$ to $730 \mathrm{~nm}$ ) while RGB acquires data in the visible spectrum (from $380 \mathrm{~nm}$ to $700 \mathrm{~nm}$ ).

The used UAVs' navigation system includes a global navigation satellite system (GNSS) receiver, with a positional accuracy of a few meters. The direct georeferencing achieved by this equipment does not follow the image's pixel resolution, enabling only an approximate location. Therefore, it is necessary to refine the external orientation through the support of tie points included in the automatic aerial triangulation processes. Ideally, these points must be uniformly distributed throughout the surveyed area, because parts that are not properly covered by GCPs are prone to more significant errors [66], since the determination of an image's exterior orientation will mainly rely on conjugate 
points between overlapping images. In general, UAV cameras are non-metric (including moving parts), and usually require a self-calibration in the bundle adjustment [67]. Correlation between exterior and interior orientation parameters (e.g., flying height and focal distance) may lead to model deformations [68], which in some cases may not be obviously detected. Not only should there be good GCPs, but independent checkpoints should also be used to verify the quality of the extracted DSM [69]. If camera pre-calibration (interior orientation parameters) is available, it should be used.

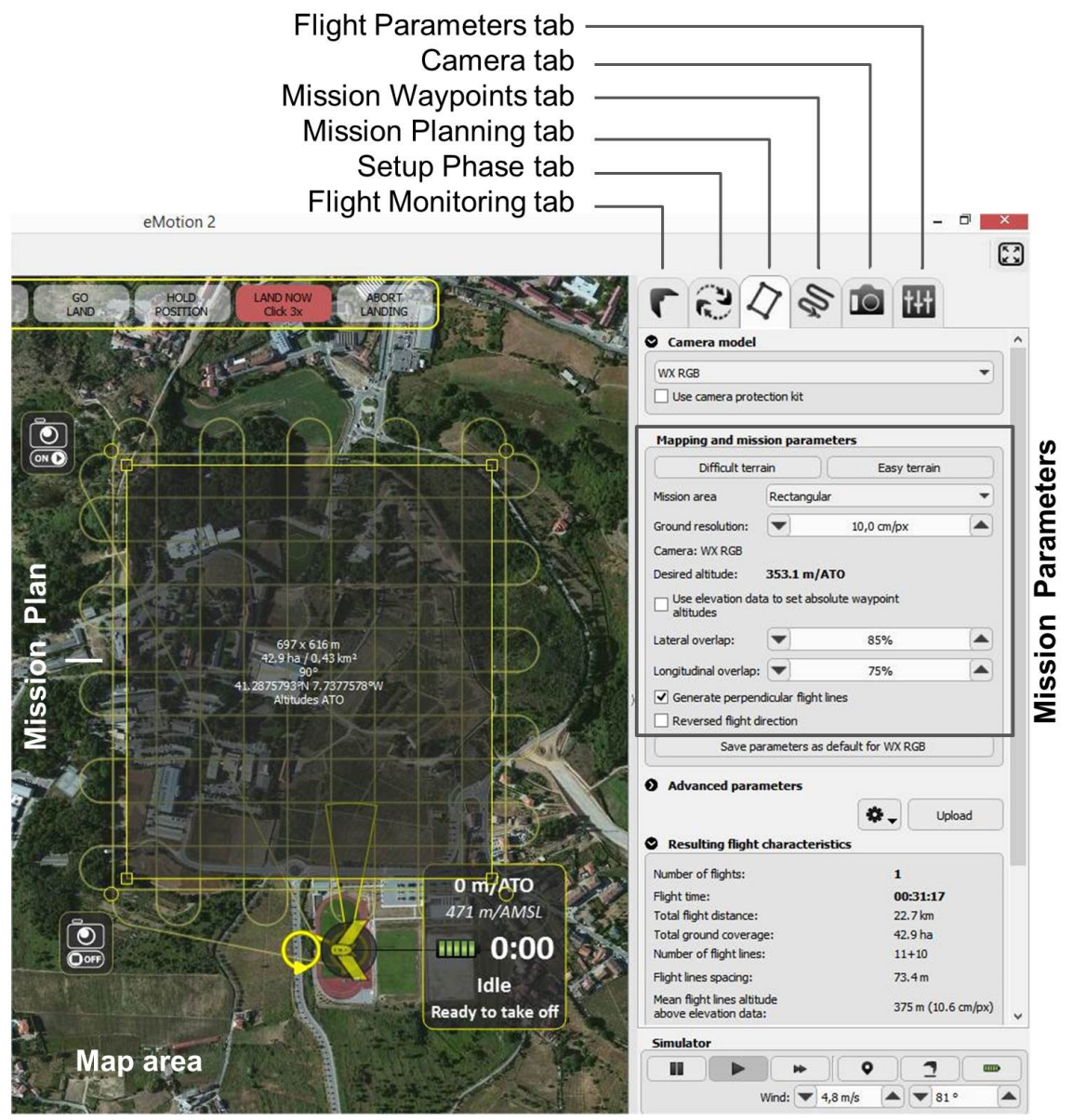

Figure 1. Planning a mission using eMotion software (senseFly SA, Lausanne, Switzerland) adjusting all the required parameters (e.g., lateral and longitudinal overlap, ground resolution).

In an urban environment, especially when road markings are present, it is relatively easy to find well-defined points that can be used as GCPs. However, in the case studies presented in this paper, it was not always possible to apply this method. As such, the option was to use panels (approximately with $1 \mathrm{~m}^{2}$ area) with center-marked crosses, placed before the flight at selected locations and fixed with metal studs. Figure 2 provides an example of a target in use, observed from two perspectives: one on the ground (Figure 2a) being surveyed by GNSS, and another presenting an UAV aerial image result (Figure $2 \mathrm{~b}$ ). 

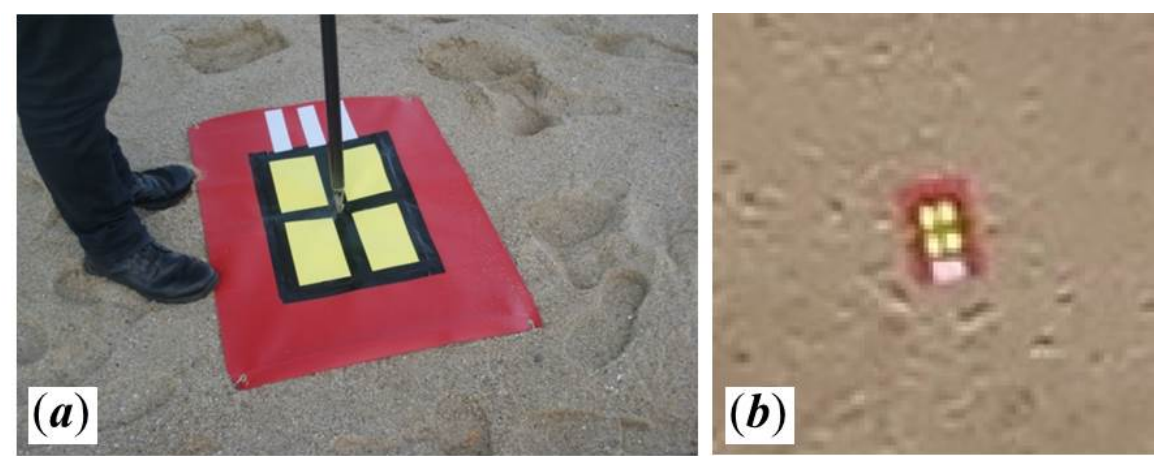

Figure 2. Example of an artificial ground control point (GCP) measuring $100 \times 65 \mathrm{~cm}$ : in (a) the ground being surveyed with a global navigation satellite system (GNSS) device placed in the middle of the marker, and in (b) an aerial image taken using an unmanned aerial system (UAS) flying at $175 \mathrm{~m}$.

\subsection{Data Processing}

Each performed flight generates large amounts of data that need to be contextualized, filtered, and analyzed in post-flight operations in order to extract information that will support the creation of knowledge for decision-making processes within forestry/agriculture (e.g., disease treatment) and preservation (e.g., natural protection) contexts.

Specific software for photogrammetric processing is required to extract information from the collected data. This type of processing usually considers the following steps. Firstly, the images are imported and the approximate internal and external orientation parameters (position-based only) provided by the navigation system are identified. Secondly, conjugate points and relative orientation blocks are generated, resulting in a 3D sparse point cloud with the approximate georeferenced location calculated from the projection centers' positions. Points with obvious errors can be eliminated from this 3D sparse point cloud and therefore from the conjugate points list. Thirdly, there is GCP insertion and refinement of the external orientation with self-calibration. Adjustments in focal length (in the principal point position), polynomial coefficients of the radial distortion $(\mathrm{K} 1, \mathrm{~K} 2, \mathrm{~K} 3)$ and tangential deformation coefficients should also be considered. Moreover, other correction parameters are not especially relevant for cameras with relatively small deformations and they can increase the risks of introducing new ones due to correlations with the external orientation parameters. Fourthly, there is the generation of a dense point cloud, obtaining a dense surface model in a grid. Finally, there is orthorectification ("true ortho" to be used in the DSM) and final mosaic creation.

\section{Case Studies}

The case studies presented in this paper consist of applying UASs in distinct fields-forestry and coastal environments-that have particular relevance in the development of socio-economic activities and in environmental sustainability. Regarding forestry, the selected UAS was applied to monitor chestnut tree health. Indeed, chestnut fruit is the main income source of the "Castanha da Padrela" region (Portugal). As for conservation and preservation, the selected UAS was applied to monitor and assess topographic changes that occurred in the Cabedelo sandspit, one of the most important and sensitive natural areas in Cabedelo (Porto, Portugal).

\subsection{Chestnut Health Monitoring}

Since the mid-1980s and mainly due to the increase in its economic importance, the area of chestnut (Castanea sativa Mill.) cultivation has been growing in Portugal. Currently, chestnut trees occupy around 36,000 hectares, of which $88 \%$ is located in northern Portugal [20]. The growing area of cultivation is clear in the "Castanha da Padrela" region (the north-eastern part of Portugal), where this case study took place. In this region, chestnut fruit is the main source of income for the local 
population [20]. However, agricultural practice intensification has favored the onset of phytosanitary problems, such as ink disease and chestnut blight. Both are considered as the main causes of chestnut tree decline [70].

Chestnut ink disease, caused by the soil-borne Phytophthora cinnamomi Rands [71] dates back to the end of the 19th century and, since then, it has been recurrently causing chestnut tree death to the present day. With respect to chestnut blight (Cryphonectria parasitica (Murr.) Barr.) [72], two decades after its first detection in Portugal, hypovirulence began to be observed in some locations. Many of the sub-populations of $C$. parasitica belong to the well characterized and specific vegetative compatibility type EU-11 —in spite of having a spectral response that can be similar to other chestnut disorders caused by, for example, abiotic factors, inadequate pruning practice, or insect defoliation-that appears only in some orchards in Italy [73]. Successful treatment depends on the way the fungus population propagates in the area of interest. In 2014 the oriental chestnut gall wasp was detected for the first time in Portugal, near Barcelos (Braga, Portugal). Scientifically known as Dryocosmus kuriphilus Yasumatsu (Hymenoptera: Cynipidae), it is considered the world's worst pest for chestnuts, and has become a serious concern for chestnut culture in Portugal due to the potential losses to fruit and timber production [74]. In three years, has rapidly spread to the most significant chestnut production areas. Many of Europe's southern and western countries have been reporting this phytosanitary issue after its accidental introduction into Piemonte (northwestern Italy), where it was found for the first time in 2002 [75].

Remote sensing techniques, such as conventional aerial photography or satellite images, are usually applied for evolution monitoring purposes. However, acquiring those images is costly, especially when the areas to be evaluated are small or there is a need to make several campaigns in relatively short periods of time [76].

In this case study, a UAS approach composed of a fixed-wing UAV (senseFly SA, Lausanne, Switzerland) was used to acquire high-resolution aerial data. This type of UAV enables the acquisition of various samples over a significant geographic area (up to $10 \mathrm{~km}^{2}$ ) in a short amount of time, especially due to the developments in sensors and their spectral and spatial resolutions [77]. As an example and when addressing the vegetation monitoring field, this kind of aerial image has been used mainly due to its advantages when compared with ground observations. Temporal and spatial high resolutions, combined with the low complexity and operation costs, make all the difference [78].

The case study area (438 ha) is located between the villages of "São João da Corveira" and "Padrela e Tazém", in Valpaços (in the north-eastern region of Portugal). For monitoring the chestnut area and recognizing the most disease-affected areas, aerophotogrammetric flights were made in three campaigns in 2014, 2015, and 2017 at an average flight altitude of $550 \mathrm{~m}$ (GSD $16 \mathrm{~cm}$ ), along six flight lines, oriented in the north-south direction. Figure 3 shows the evolution of a small part of the case study area, over time. Later on, these aerial images-both in color (RGB) and near-infrared (NIR) - were used to compare the evolution in consecutive campaigns. RGB images acquired in 2006 by the Portuguese Forest Authority for the National Forest Inventory, with one-meter GSD [79], were also included in the case study in order to extend the analyzed period of time. After the flights, image orthorectification and geocoding were performed based on natural GCPs directly identified in the images. 

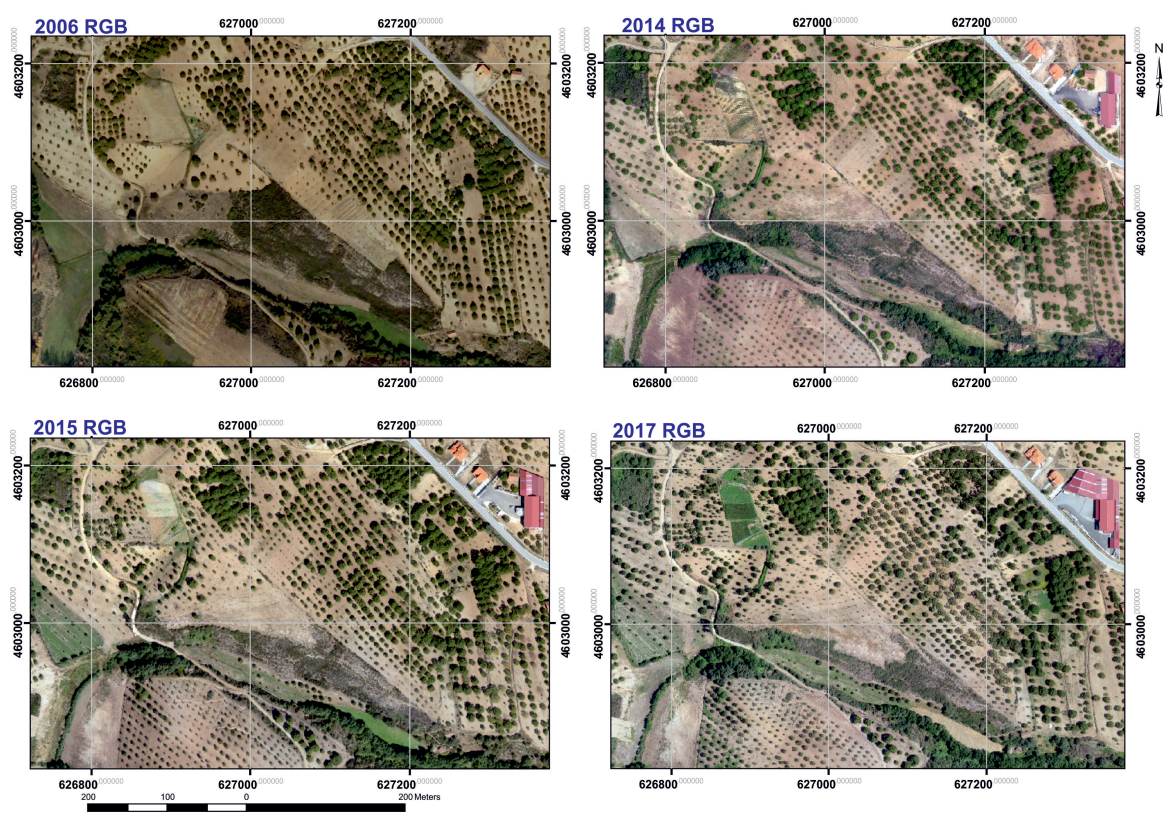

Figure 3. Temporal evolution of a portion of the study area in each campaign. RGB: red-green-blue.

\subsubsection{Considerations about Surveys of Chestnut Trees}

Photographic keys relating to chestnut trees with different physiological conditions were developed for photo interpretation purposes. Moreover, field data acquired during the campaigns were compared with the acquired aerial images (Figure 4), which in turn were processed using Pix4Dmapper software (Pix4D SA, Lausanne, Switzerland).

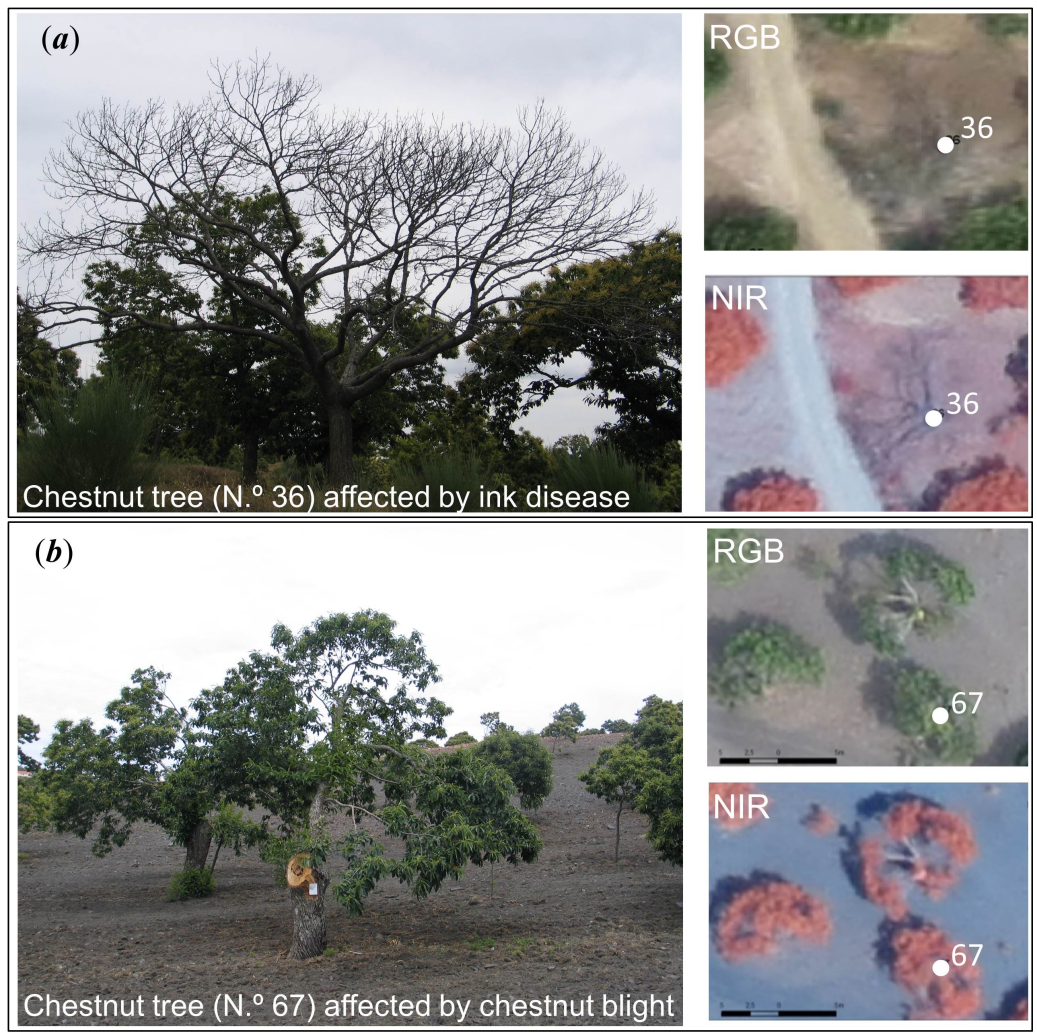

Figure 4. Chestnut trees affected by (a) ink disease and (b) chestnut blight. The same trees are represented in color and infrared aerial photographs. NIR: near-infrared. 
A geographic information system (GIS) was used for distributing 438 circular plots in the case study area (500 $\mathrm{m}^{2}$ area, each), by using a systematic distribution of $100 \times 100 \mathrm{~m}^{2}$, corresponding to a 1-ha grid. Color and NIR orthorectified aerial photographs obtained in the different campaigns were used in the GIS environment to determine differences in the chestnut canopy between three consecutive campaigns (2006-2014, 2014-2015 and 2015-2017). Canopy cover index (CI) (Equation (1)) considers the ratio between the area covered by the chestnut canopy and each plot's surface $\left(500 \mathrm{~m}^{2}\right)$. This ratio represents ranges from 0 to 100 . The same procedure was adopted in all campaigns.

$$
C I=\frac{C A}{P A} \times 100
$$

In Equation (1), CA represents the plot's canopy area and PA the plot's area $\left(500 \mathrm{~m}^{2}\right.$ in this specific case). To estimate health of the chestnut trees, as well as mortality and new plantation areas, a Student's t-distribution was used. A sampling error with a 95\% confidence level was considered. To assess the geographical evolution of vitality, methods to estimate parameters based on attributes observed in neighbor points were used [80]. These methods are used to explain the spatial structured phenomena (such as forest diseases) because they do not have a random distribution.

The existence of spatial correlation between georeferenced random variables correlation (that depends on the distance between points, which tends to decrease with distance), can be found using geostatistical methods [81]. These methods use specific observations for a single regionalized variable of interest, $\mathrm{Z}(\mathrm{xi})$, referred to a set of points [82] of the study area (univariate methods). Alternatively, auxiliary regionalized variables, whose values can contribute to the improvement of estimates of the main interest variable, can be used to provide the correlation rate.

The behavior of regional variables in the interpolation, $\mathrm{Z}(\mathrm{xi})$, whose spatial continuity could be modeled by a semivariogram represented by Equation (2), was observed. The chestnut growth (CG) for each multi-temporal survey was used as the variable in the geostatistical approach. The model does not use negative or null values. In this sense, the CG was converted into a scale ranging from 1 to 20. The higher the value, the better the tree's health condition.

$$
C I=\frac{1}{2} \times E\left[Z\left(x_{i}+h\right)-Z\left(x_{i}\right)\right]^{2}
$$

The results obtained in two consecutive campaigns were used to evaluate the difference between CIs. For example, the results obtained by using the 2006 and 2014 campaign images were used to estimate CG during that period of time. The CG (Equation (3)) reflects the predictable growth (where $C G>0$ ), but also the chestnut decline (if $C G<0$ ). A $5 \%$ chestnut growth rate was admitted for the 8-year period. This is the predictable chestnut trees development considering the soil and climatic conditions of the case study area [70]. For the other two periods (2014-2015 and 2015-2017), a 0\% minimum growth rate was considered [70].

$$
C G(14-06)=C I_{14}-C I_{16}
$$

where:

$$
\begin{aligned}
& \mathrm{CG}-\text { Chestnut growth (\%) } \\
& \mathrm{CI}_{16} \text {-Canopy cover index in } 2006 \\
& \mathrm{CI}_{14} \text {-Canopy cover index in } 2014
\end{aligned}
$$

\subsubsection{Results and Discussion}

Table 1 presents the CI results for all the campaigns, with different values for each one. Regarding the 2006-2014 period, CI has significant differences and an important decline can be noticed in 55\% of the plots. As for the 2014-2015 period, the decline was even more pronounced, occurring in $60 \%$ of the chestnut plots. Between 2015 and 2017, the decline occurred in 35\% of the chestnut plots. As it can be observed in Table 2, new plantations were made in forestry areas, abandoned areas, 
or in soils with less profitable cultures (cereals, pastures, potatoes, etc.). These practices positively influenced the results obtained in the latter analyzed period (2015-2017). A significant contribution for this overall result was given by new plantations that increased the total chestnut area by $40 \%$. The $247 \pm 10$ ha area in 2006 now measures $347 \pm 14$ ha (Table 2). For this reason, the decline can also be related to inadequate soils for such a demanding culture as Castanea sativa [83] .

Table 1. CI and chestnut area for the period of the study (2006-2017). The sampling error is according to Student's t-distribution. The average values with $\left(^{*}\right)$ are significantly equal.

\begin{tabular}{lcccc}
\hline \multicolumn{1}{c}{ Parameter/Year } & $\mathbf{2 0 0 6}$ & $\mathbf{2 0 1 4}$ & $\mathbf{2 0 1 5}$ & $\mathbf{2 0 1 7}$ \\
\hline Canopy cover index (CI) & $21.6 \pm 2.5 \%\left(^{*}\right)$ & $19.5 \pm 1.8 \%$ & $22.2 \pm 2.0 \%\left(^{*}\right)$ & $25.9 \pm 2.1 \%$ \\
CI minimum & 5 & 5 & 0 & 0 \\
Canopy cover per hectare (CC/ha) & $2160 \pm 250 \mathrm{~m}^{2}\left(^{*}\right)$ & $1950 \pm 180 \mathrm{~m}^{2}$ & $2220 \pm 200 \mathrm{~m}^{2}\left(^{*}\right)$ & $2590 \pm 210 \mathrm{~m}^{2}$ \\
CI maximum & 100 & 90 & 90 & 90 \\
Sampling error (SE $\%)$ for CC & $11.7 \%$ & $9.2 \%$ & $9.2 \%$ & $8.2 \%$ \\
Total area (SE $\%=4 \%)$ & $438 \pm 18$ ha & $438 \pm 18$ ha & $438 \pm 18$ ha & $438 \pm 18$ ha \\
Chestnut area $(\mathrm{SE} \%=4 \%)$ & $247 \pm 10$ ha & $303 \pm 12$ ha & $295 \pm 12$ ha & $347 \pm 14$ ha \\
\hline
\end{tabular}

Table 2. Chestnut area and chestnut decline affecting the whole study area (438 ha).

\begin{tabular}{|c|c|c|c|c|c|c|c|}
\hline & 2006 & 2014 & & 2015 & & 2017 & \\
\hline Other cultures & $191(44 \%)$ & $135(31 \%)$ & & $143(33 \%)$ & & $91(21 \%)$ & \\
\hline Chestnut area (ha) & $247(56 \%)$ & $303(69 \%)$ & & $295(67 \%)$ & & $347(79 \%)$ & \\
\hline Chestnut decline & & & $135(55 \%)$ & & $182(60 \%)$ & & $104(35 \%)$ \\
\hline Chestnut growth & & & $112(45 \%)$ & & $121(40 \%)$ & & $191(65 \%)$ \\
\hline Chestnut area variation & & & $\begin{array}{c}{[303-247]} \\
(18 \%)\end{array}$ & & $\begin{array}{c}{[295-303]} \\
(-3 \%)\end{array}$ & & $\begin{array}{c}{[347-295]} \\
(15 \%)\end{array}$ \\
\hline Total (ha) & 438 & 438 & 247 & 438 & 303 & 438 & 295 \\
\hline
\end{tabular}

The geostatistical approach allowed for the detection of the three important affected areas (Figure 5). The decline in foci detected in the 2006-2014 period worsened in the 2014-2015 period. However, an improvement in the health condition of chestnut trees was observed in the 2015-2017 period. These results are in accordance with Table 2.

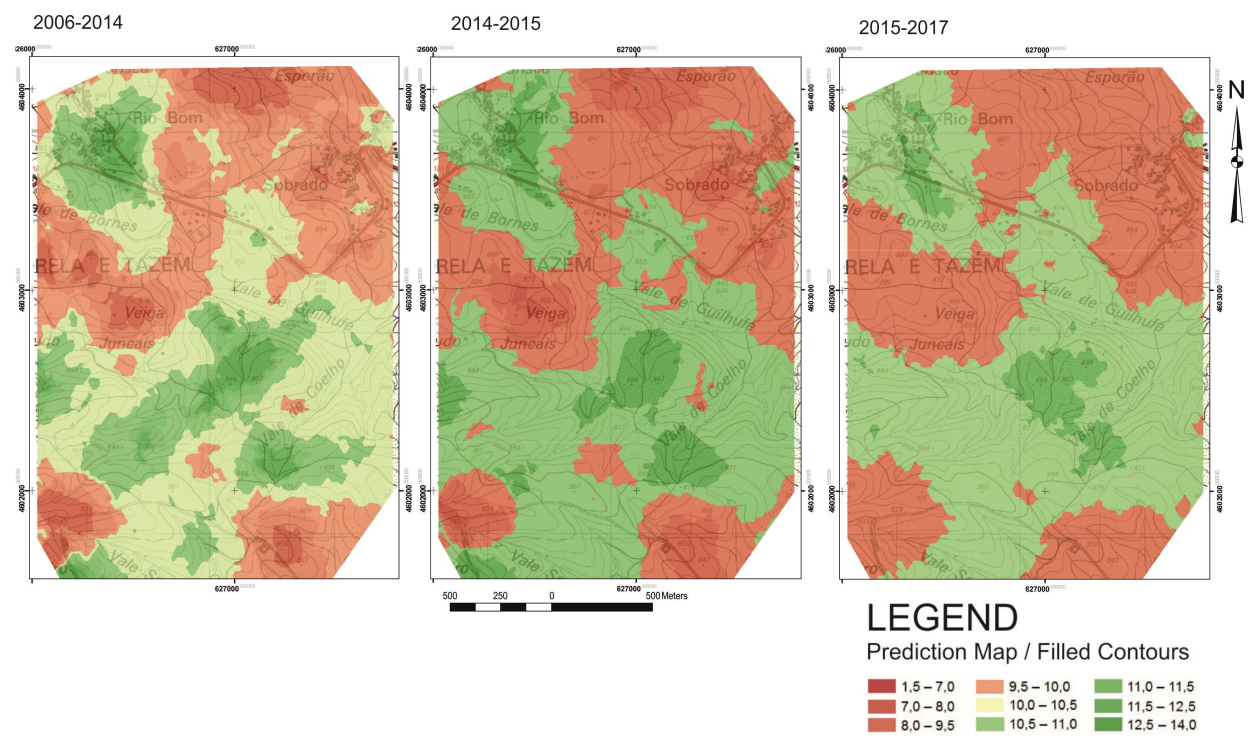

Figure 5. Chestnut growth (CG) and decline for the 2006-2014, 2014-2015, and 2015-2017 periods. CG was converted into a scale ranging from 1 to 20 . The higher the value, the better the tree's health condition. 
Moreover, these results also demonstrate that RGB and RE/NIR aerial imagery obtained by UAS is a cost-effective alternative to other remote sensing platforms, as they are reliable for monitoring chestnut tree health, allowing mapping affected areas quickly and accurately, namely in:

- detecting chestnut ink disease symptoms [84];

- monitoring tree canopy cover decline by means of multi-temporal analysis (assess chestnut blight presence);

- providing the means for evaluating gall wasp biological control strategy effectiveness.

Furthermore, this approach also demonstrated to be an effective tool for classifying soil occupation, detecting areas of interest for new cultures and evaluating new plantations.

This study also confirmed interest in chestnut culture, as it showed the new plantations that increased the area of chestnut cultivation from 247 ha in 2006, to 347 ha in 2017 (a $40 \%$ greater chestnut area in the case study area in the referred time period). The last period studied (2015-2017) shows a positive value on CG. This growth is related to the new practices used for controlling biotic agents. In fact, the lower soil tillage to reduce chestnut ink disease and the application of hypovirulence strains to control chestnut blight may be directly responsible for this improvement [85].

Lastly, using this approach (when compared with field observations) enables the recognition and quantification of the chestnut tree decline, disease dispersion and the respective most-affected areas. It was also possible to evaluate the decline of the chestnut tree at a substantially lower cost compared to other field surveys or manned aircraft-based images [86]. In Portugal, chestnut is currently facing severe climatic conditions characterized by heat and drought stresses with important consequences for species' health. Thus, it is convenient to model yield forecast and species area redistribution according to climatic constraints.

Due to the similar aerial imagery behavior presented by other species with high economic impact in Portugal (Quercus suber L. and Olea europea L.), the obtained results allowed us to conclude that it could be possible to adapt the present methodology to be applied in those species.

\subsection{Cabedelo Sandspit Variation Assessment}

The Cabedelo sandspit is responsible for the preservation of ecosystems and for the protection of the sand area. Its dunes are crucial for preserving the dwellings situated on the coastline and those of the local population, as it prevents the Douro River banks being reached by waves. Moreover, there are financial interests in terms local business activities being run in the Douro River estuary navigation channel, and these are highly dependent upon the sandspit conservation [87].

At present, the Cabedelo sandspit has an approximate size of $800 \times 400 \mathrm{~m}$ and is well known for its frequent changes in position and shape. Before the construction of a detached jetty, the sandspit morphodynamics were related to extreme river flow, sea turbulence, and wind. After the construction, the sandspit shape was stabilized and an increase in its area and volume was observed as well [88]. Figure 6 shows orthoimages of different periods that show the large variations in shape and location, as well as the stabilization that occurred after 2006. Through Figure 6 it is possible to obtain a multi-temporal view of the morphological dynamics of the case study area.
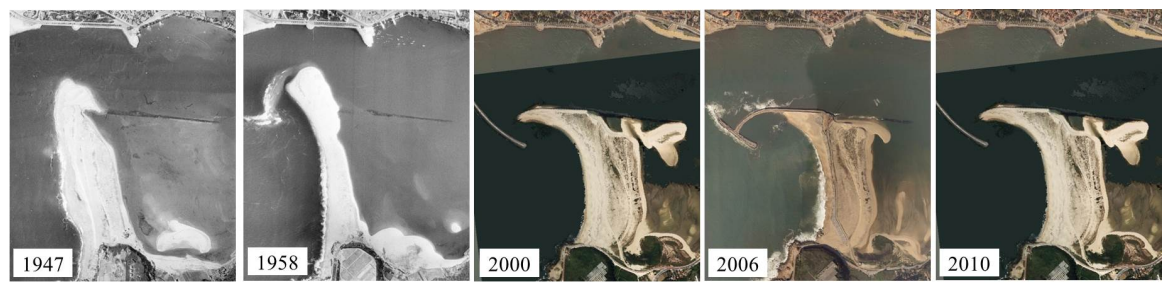

Figure 6. Orthoimages of the sandspit in five different periods (images provided by aerial national mapping agency aerial photography archives). 
A monitoring program has been developed by the University of Porto, based on GNSS land surveys as well as photogrammetric surveys, using the digital camera ZI-DMC-I $[44,88]$. Some conventional aerial photography campaigns were carried out, covering a coastline extension of $15 \mathrm{~km}$. Aerial photography would not be affordable just for the Cabedelo sandspit monitoring. The best solution for fast, frequent, and cost-effective monitoring surveys of this area is the use of a UAS. The first survey was carried out in 2013.

In the next few subsections the changes that occurred between the three multi-temporal series are analyzed and quantified. Several aspects related to the accuracy of the extracted DSM are also analyzed as they may strongly affect the alteration assessment. Although GCPs were not exactly the same in number, eight of them were placed at the same location in all flights, using a global positioning system (GPS) navigation device. Their location was rigorously surveyed using differential GPS, in real-time kinematic (RTK) mode.

\subsubsection{Considerations about Surveys in Sand Areas}

The main purpose of UAS surveys in coastal areas is to assess topographic changes in sand volume. For this reason, geometrical accuracy of the resulting surface models and orthoimages are fundamental for the analysis. Sandy areas pose several challenges that must be carefully analyzed First, there is the need for accurate GCPs, which must be well defined on both the ground and on the images. Unlike in built-up areas, the natural environments of coastal areas do not provide such points, so artificial marks must be previously placed on the ground (Section 3.1).

For this case study, vertical checkpoints are most important since they allow for control of sand volume variation. These are relatively simple to acquire, since surveys of RTK GNSS can be very fast and points do not need to be marked. For a vertical checkpoint of coordinates $(E, N, h)$, residuals $(\Delta h)$ are calculated by subtracting the height measured by GNSS $\left(h_{G N S S}\right)$ and the height interpolated from the DSM, on coordinates $(E, N)$, using bi-linear interpolation $\left(h_{D S M}\right)$ (Equation (4)). The overall accuracy is given by the root-mean-square error (RMSE), for $n$ observed checkpoints, as in Equation (5). The mean and the standard deviation can also be determined to assess if some systematic trend may exist in the data.

$$
\begin{gathered}
\Delta h_{i}=h_{i, G N S S}-h_{D S M}\left(E_{i}, N_{i}\right), i=1, \ldots, n \\
R M S_{h}=\sqrt{\frac{\sum_{i=1}^{n} \Delta h_{i}^{2}}{n}}
\end{gathered}
$$

Beside these challenges, others that are typical of sandy areas must be considered to obtain success in applying UAS in this kind of environment. These challenges include, for example, the lack of patterns that sand may have, reducing the quality of the conjugate points obtained by stereo matching. This is especially the case when the sun is high, which may result in too-bright images. It is preferable to perform surveys on cloudy days or in the early morning, when the sun is low [89]. Another difficulty, which is combined with sun illumination and the tide, is the requirement of relatively low wind. This is not the regular situation in the Portuguese coastal zone [90], which makes the availability of adequate moments for the surveys relatively difficult. In this case study, there was also the need to combine the flight time with a low tide. The first flight made in 2013 was performed in the early morning of a cloudy day. In fact, it gave the best vertical accuracy.

Finally, it was possible to verify that waves also pose some difficulties. Although low tides were chosen, with instantaneous sea level height at $-1 \mathrm{~m}$ or below, waves may be present in heights of up to 1 or even $2 \mathrm{~m}$. The extracted DSM has very poor quality in these areas. For that reason, contour comparisons between different campaigns were made for an elevation of $2 \mathrm{~m}$ above the sea level. 


\subsubsection{Results and Discussion}

The first survey was conducted in 2013 with a smaller resolution camera (when compared with the two more recent campaigns) coupled on senseFly Swinglet (senseFly SA, Lausanne, Switzerland) with a GSD of $4.5 \mathrm{~cm}$. The other two surveys were performed in 2015 and 2017, using the UAV SenseFly eBee (senseFly SA, Lausanne, Switzerland) and a GSD of $5.2 \mathrm{~cm}$. This latter GSD meant that for practical purposes the resolution was slightly decreased, while keeping the standards for the monitoring objectives.

The first campaign was performed for experimental reasons and included detailed quality control analysis, which is described in [45]. The image orientation process by bundle adjustment was done in Agisoft Photoscan (Agisoft LLC, St. Petersburg, Russia) and provided residuals similar to the GSD, both in planimetry and altimetry. The main concern, especially because the models are intended to rigorously assess height changes, was with vertical accuracy. Although the bundle adjustment may be good in terms of the control points, in areas not so well covered by GCPs the model may have deformations, especially if many adjustment parameters are used. As referred to before, an independent verification with altimetric checkpoints is important to verify that deformations do not occur. Elevation checkpoints were obtained by differential GNSS at the same time as GCP collection, with care taken to choose places where sand had not been moved and without leaving the pole in the sand. At least 100 checkpoints-well distributed throughout the area-were to be acquired, per survey.

The second campaign-done in May 2015-was performed during the breeding season of some birds, such as sand plovers, which limited the access to some areas. Therefore, a smaller number of checkpoints were collected. However, the distribution was reasonably complete. Table 3 presents data from the three campaigns, namely their characteristics and the accuracy analysis results.

The vertical errors found on the independent check points (ICP) have positive and negative values, and approximately follow a Gaussian distribution with an average close to zero [45]. This fact reveals that no systematic trends exist in the surface models. There is an error propagation to the calculated volumes but this is not as significant as if a systematic vertical trend were to exist on the surface.

Table 3. Cabedelo sandspit campaigns $(2013,2015,2017)$ characteristics and analysis results. UAV: unmanned aerial vehicle; GSD: ground sample distance.

\begin{tabular}{lccccc}
\hline $\begin{array}{l}\text { Date and Start } \\
\text { Time (h:min) }\end{array}$ & $\begin{array}{c}\text { UAV/Camera } \\
\text { Resolution }\end{array}$ & GSD & $\begin{array}{c}\text { \# Images } \\
\text { Used }\end{array}$ & $\begin{array}{c}\text { GCPs Total/3D } \\
\text { RMS }\end{array}$ & $\begin{array}{c}\text { ICPs } \\
\text { Total/RMS }\end{array}$ \\
\hline 22 July 2013 07:22 & Swinglet/12 Mp & $4.5 \mathrm{~cm}$ & 308 & $11 / 12.8 \mathrm{~cm}$ & $114 / 4.6 \mathrm{~cm}$ \\
06 May 2015 10:55 & eBee/16 Mp & $5.2 \mathrm{~cm}$ & 204 & $8 / 3.0 \mathrm{~cm}$ & $34 / 6.3 \mathrm{~cm}$ \\
29 March 2017 11:15 & eBee/16 Mp & $5.2 \mathrm{~cm}$ & 196 & $9 / 4.2 \mathrm{~cm}$ & $146 / 7.1 \mathrm{~cm}$ \\
\hline
\end{tabular}

DSMs were generated for the tree campaigns, which enabled the assessment of differences due to the sand movements. Figure 7a shows a color-coded image of the 2017 campaign DSM. Figure 7b shows the hill shaded image of the DSM, together with the corresponding contours of height of $2 \mathrm{~m}$. The 2-m contours of the 2013 and 2015 campaigns DSMs are overlaid on top of the 2017 DSM, in different colors To perceive the sand accumulation, profiles were traced in the place of largest separation between the contours, along the steepest slope (A and B). These profiles are represented in Figure 8. 

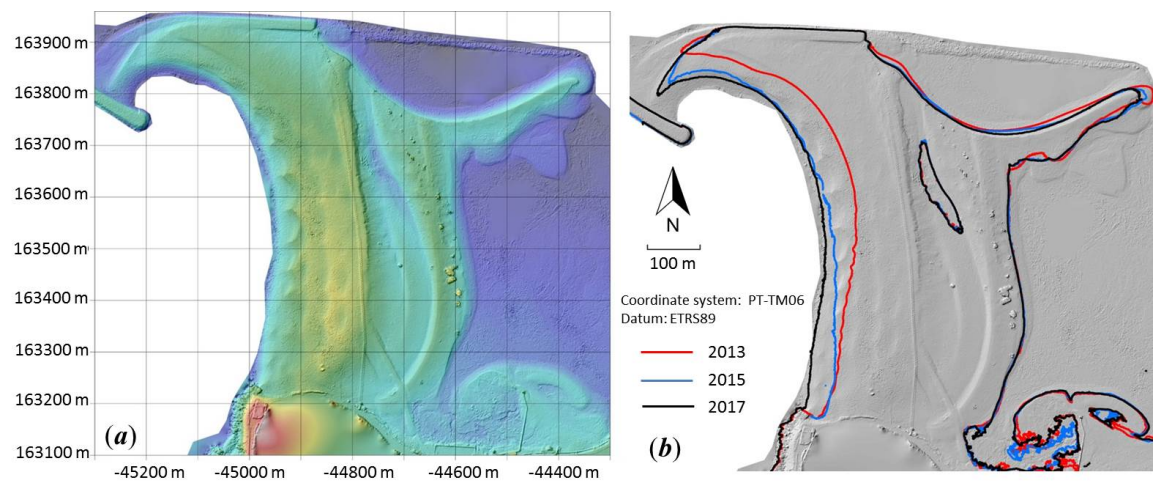

Figure 7. Differences in Cabedelo sandspit due to the sand movements: (a) colour coded DSM of 2017 and (b) hillshaded DSM with contours of the 2013 and 2015 DSMs overlaid.
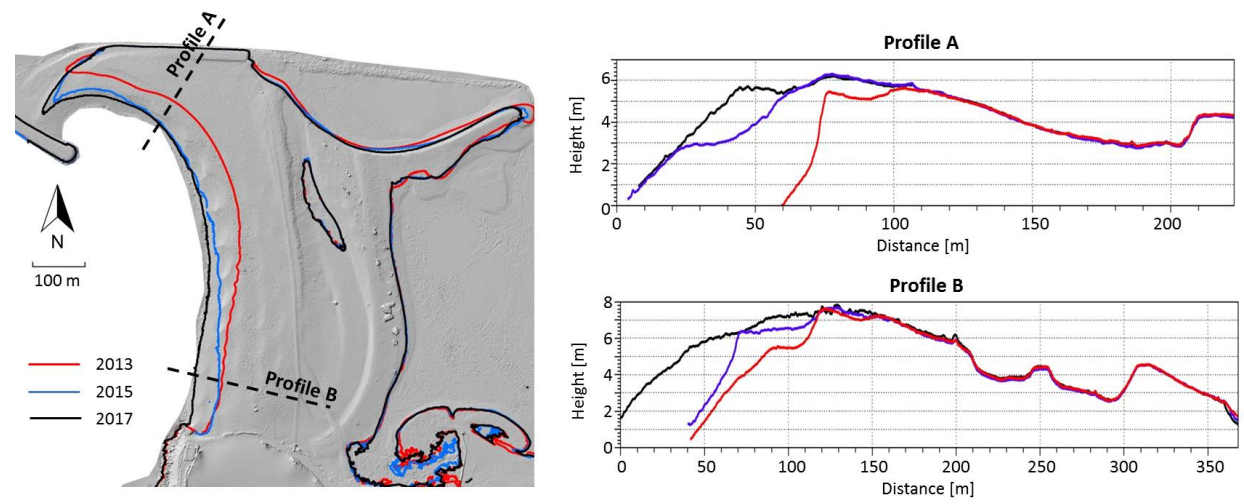

Figure 8. Profiles along the steepest slope, in the part of largest sand increase for the three epochs: red for 2013, blue for 2015, and black for 2017. Profile A had larger increase from 2013 to 2015, while in profile B the largest increase was from 2015 to 2017.

The main change occurred from 2013 to 2015, with a large accumulation of sand (around $60 \mathrm{~m}$ ) in the northern and central parts of the sandspit, facing the sea. From 2015 to 2017 there was an accumulation in the southern part of around $40 \mathrm{~m}$. The models were subtracted to calculate the volume difference between consecutive DSMs as the sum of vertical prisms. The largest volume calculated for the increased area facing the sea was of 170,000 cubic meters (volumes above height zero), between 2013 and 2015. The increase from 2015 and 2017 was of approximately 60,000 cubic meters. Sand accumulation that was observed with the DSMs acquired in the successive UAV flights is due to the detached breakwater built in the area. Its aim is to disperse wave energy and fix the sandspit to facilitate boat navigation in the Douro river mouth. As expected [87], there is an accumulation of sand in the sandspit. Its increase rate (that can be measured from the UAV data) can help in taking measures for the coastal engineering management of the area.

An additional remark on the profile analysis is the noise effect present on the DSMs. The first DSM (2013) was smoother than the other two, especially the one from 2017, which may be explained because it was done on a cloudy day. In the other two, especially that of 2017, there was sun and they were done in the middle of the morning: images were much brighter and with less contrast and patterns for the matching process. This is concordant with the lower vertical accuracy (ICP RMS ${ }_{h}$ in Table 3). In any case, detection and quantification of the differences between the three DSMs was possible due to the sand deposition by the sea. This study confirms the feasibility of this methodology for change assessment in sandy beaches. Many studies recently published reveal that it is being regularly used to assess changes in critical areas. From the experience obtained with this study, which involved a 
relatively sparse dataset along time with an average revisit period of two years, more frequent surveys would be needed to perceive the continuity of the change process. Some limitations were found due to environmental issues, such as the frequent strong winds in the Portuguese Atlantic coast. Another important constraint was due to logistic effects, because of the need for signalized ground control points. Although flight times were as short as $30 \mathrm{~min}$, several hours were needed to place the signals, survey their coordinates with GNSS, and collect them back. This requirement makes surveys rather time consuming and not so simple to implement with higher frequencies.

A very important improvement can be achieved with UAVs equipped with precise GNSS equipment, working in RTK or post processing kinematic (PPK) mode. Surveys without ground control points, keeping a suitable accuracy [14,91], can be conducted, allowing for much simpler logistics of data collection. With smaller requirements for field work, a more frequent observation would be possible, allowing not only for change assessment and quantification, but also for a better perception of periodical phenomena, with regular data collection before and after winter seasons.

\section{Conclusions}

This work presents two applications of unmanned aerial systems, one of the most recent remote sensing technologies. In the first case study, RGB and NIR high-resolution aerial images were used to monitor the evolution of a chestnut tree area over time. The feasibility of this approach was demonstrated by comparing the results with ground true data. A good agreement was found. Tree canopies, computed in both RGB and NIR high-resolution images, were also used to detect the coverage's evolution. In that way, it was possible to correlate that evolution (growth or decline) with biotic and abiotic factors. Thus, UAS-based methods allow us to detect and fight the major issues affecting chestnut trees.

The second case study presented in this work focuses on the monitoring of the Cabedelo sandspit. In sensitive ecosystems (like this one), the use of UAVs avoids having to walk the terrain, which usually leads to severe damage which, for instance, occurs with ground vehicle tracks. It was possible to detect a significant change between 2013 and 2015, with a large build-up of sand in the northern and central parts of the sandspit. In the study's most recent period (2015-2017), there was an accumulation in the southern part of the sandspit. Within the total period analyzed, the sandspit's total volume increased by more than 200,000 cubic meters of sand.

The continuity of both studies will support better knowledge and understanding in assessing the effects of corrective measures that have been applied by chestnut tree producers in the last years, and a better understanding of the dynamics and coastal protection works performed in the Cabedelo sandspit study area.

The UAS may be considered a well-suited configurable tool which is fairly flexible for application in such distinct areas as forest and coastal environments. Moreover, they constitute a cost-effective and non-invasive form of technology capable of covering considerably-sized areas in a single flight, supporting different sensors within their payload. Currently, UASs continue to evolve, offering new opportunities and presenting new challenges.

Acknowledgments: This work was financed by the European Regional Development Fund (ERDF) through the Operational Programme for Competitiveness and Internationalisation-COMPETE 2020 under the PORTUGAL 2020 Partnership Agreement, and through the Portuguese National Innovation Agency (ANI) as a part of project "PARRA-Plataforma integrAda de monitoRização e avaliação da doença da flavescência douRada na vinhA" ( $N^{\mathrm{O}}$ 3447), as well as the ERDF and North 2020-North Regional Operational Program, as part of the project “INNOVINE\& WINE-Vineyard and Wine Innovation Platform" (NORTE-01-0145-FEDER-000038).

Author Contributions: Luís Pádua, Jonáš Hruška, José Bessa and Telmo Adão were involved in the paper writing and in the experimental data processing. Emanuel Peres, António M. R. Sousa and Joaquim J. Sousa designed and supervised paper development. Regarding the chestnut trees health monitoring, data collection was carried out by Luís M. Martins, João P. Castro, Luís Pádua and Joaquim J. Sousa. Luís Pádua and Jonáš Hruška also carried out the photogrammetric processing. Results interpretation was performed by Luís M. Martins and João P. Castro. Regarding the Cabedelo sandspit, data acquisition was carried out by José A. Gonçalves and Joaquim J. Sousa. The former author was also in charge of photogrammetric processing and results interpretation/discussion. 
Conflicts of Interest: The authors declare no conflict of interest.

\section{References}

1. Ermacora, G.; Toma, A.; Rosa, S.; Bona, B.; Chiaberge, M.; Silvagni, M.; Gaspardone, M.; Antonini, R. A Cloud Based Service for Management and Planning of Autonomous UAV Missions in Smart City Scenarios. In Proceedings of the Modelling and Simulation for Autonomous Systems: First International Workshop (MESAS), Rome, Italy, 5-6 May 2014; Hodicky, J., Ed.; Revised Selected Papers; Springer International Publishing: Cham, Switzerland, 2014; pp. 20-26.

2. Oleire-Oltmanns, S.; Marzolff, I.; Peter, D.K.; Ries, B.J. Unmanned Aerial Vehicle (UAV) for Monitoring Soil Erosion in Morocco. Remote Sens. 2012, 4, 3390-3416.

3. Jenkins, D.; Vasigh, B. The Economic Impact of Unmanned Aircraft Systems Integration in the United States. Available online: http://www.auvsi.org/auvsiresources/economicreport (accessed on 23 December 2017).

4. Pádua, L.; Vanko, J.; Hruška, J.; Adão, T.; Sousa, J.J.; Peres, E.; Morais, R. UAS, sensors, and data processing in agroforestry: A review towards practical applications. Int. J. Remote Sens. 2017, 38, 2349-2391.

5. Matese, A.; Toscano, P.; Di Gennaro, S.F.; Genesio, L.; Vaccari, F.P.; Primicerio, J.; Belli, C.; Zaldei, A.; Bianconi, R.; Gioli, B. Intercomparison of UAV, aircraft and satellite remote sensing platforms for precision viticulture. Remote Sens. 2015, 7, 2971-2990.

6. Atzberger, C. Advances in Remote Sensing of Agriculture: Context Description, Existing Operational Monitoring Systems and Major Information Needs. Remote Sens. 2013, 5, 949-981.

7. Mirijovský, J.; Langhammer, J. Multitemporal Monitoring of the Morphodynamics of a Mid-Mountain Stream Using UAS Photogrammetry. Remote Sens. 2015, 7, 8586-8609.

8. Tanteri, L.; Rossi, G.; Tofani, V.; Vannocci, P.; Moretti, S.; Casagli, N. Multitemporal UAV Survey for Mass Movement Detection and Monitoring. In Advancing Culture of Living with Landslides: Volume 2 Advances in Landslide Science; Mikos, M., Tiwari, B., Yin, Y., Sassa, K., Eds.; Springer International Publishing: Cham, Switzerland, 2017; pp. 153-161.

9. Jomaa, I.; Auda, Y.; Abi Saleh, B.; Hamzé, M.; Safi, S. Landscape spatial dynamics over 38 years under natural and anthropogenic pressures in Mount Lebanon. Landsc. Urban Plan. 2008, 87, 67-75.

10. Niethammer, U.; James, M.R.; Rothmund, S.; Travelletti, J.; Joswig, M. UAV-based Remote Sensing of the Super-Sauze Landslide: Evaluation and Results. Eng. Geol. 2012, 128, 2-11.

11. Cho, J.; Lim, G.; Biobaku, T.; Kim, S.; Parsaei, H. Safety and Security Management with Unmanned Aerial Vehicle (UAV) in Oil and Gas Industry. Procedia Manuf. 2015, 3, 1343-1349.

12. Dooly, G.; Omerdic, E.; Coleman, J.; Miller, L.; Kaknjo, A.; Hayes, J.; Braga, J.; Ferreira, F.; Conlon, H.; Barry, H.; et al. Unmanned Vehicles for Maritime Spill Response Case Study: Exercise Cathach. Mar. Pollut. Bull. 2016, 110, 528-538.

13. Funaki, M.; Higashino, S.I.; Sakanaka, S.; Iwata, N.; Nakamura, N.; Hirasawa, N.; Obara, N.; Kuwabara, M. Small Unmanned Aerial Vehicles for Aeromagnetic Surveys and Their Flights in the South Shetland Islands, Antarctica. Polar Sci. 2014, 8, 342-356.

14. Turner, I.L.; Harley, M.D.; Drummond, C.D. UAVs for Coastal Surveying. Coast. Eng. 2016, 114, $19-24$.

15. Candiago, S.; Remondino, F.; De Giglio, M.; Dubbini, M.; Gattelli, M. Evaluating Multispectral Images and Vegetation Indices for Precision Farming Applications from UAV Images. Remote Sens. 2015, 7, 4026-4047.

16. Lisein, J.; Pierrot-Deseilligny, M.; Bonnet, S.; Lejeune, P. A Photogrammetric Workflow for the Creation of a Forest Canopy Height Model from Small Unmanned Aerial System Imagery. Forests 2013, 4, 922-944.

17. Colomina, I.; Molina, P. Unmanned Aerial Systems for Photogrammetry and Remote Sensing: A Review. ISPRS J. Photogramm. Remote Sens. 2014, 92, 79-97.

18. Jia, Y.; Su, Z.; Shen, W.; Yuan, J.; Xu, Z. UAV Remote Sensing Image Mosaic and Its Application in Agriculture. Int. J. Smart Home 2016, 10, 159-170.

19. Prabhakar, M.; Prasad, Y.G.; Rao, M.N. Remote Sensing of Biotic Stress in Crop Plants and Its Applications for Pest Management. In Crop Stress and Its Management: Perspectives and Strategies; Venkateswarlu, B., Shanker, A.K., Shanker, C., Maheswari, M., Eds.; Springer: Dordrecht, The Netherlands, 2012; pp. 517-545.

20. De Estatística, I.N. Estatísticas Agrícolas; Instituto Nacional de Estatística Statistics Portugal: Lisbon, Portugal, 2016. 
21. Chen, J.S.; Li, L.; Wang, J.Y.; Barry, D.A.; Sheng, X.F.; Gu, W.Z.; Zhao, X.; Chen, L. Water resources: Groundwater maintains dune landscape. Nature 2004, 432, 459-460, doi:10.1038/432459a.

22. Mancini, F.; Dubbini, M.; Gattelli, M.; Stecchi, F.; Fabbri, S.; Gabbianelli, G. Using Unmanned Aerial Vehicles (UAV) for High-Resolution Reconstruction of Topography: The Structure from Motion Approach on Coastal Environments. Remote Sens. 2013, 5, 6880-6898.

23. Pettorelli, N.; Laurance, W.F.; O’Brien, T.G.; Wegmann, M.; Nagendra, H.; Turner, W. Satellite remote sensing for applied ecologists: Opportunities and challenges. J. Appl. Ecol. 2014, 51, 839-848.

24. Ponti, M.P. Segmentation of Low-Cost Remote Sensing Images Combining Vegetation Indices and Mean Shift. IEEE Geosci. Remote Sens. Lett. 2013, 10, 67-70.

25. Zhang, C.; Kovacs, J.M. The Application of Small Unmanned Aerial Systems for Precision Agriculture: A Review. Precis. Agric. 2012, 13, 693-712.

26. Whitehead, K.; Hugenholtz, C.H. Remote sensing of the environment with small unmanned aircraft systems (UASs), part 1: A review of progress and challenges. J. Unman. Veh. Syst. 2014, 2, 69-85.

27. Fraser, R.H.; Olthof, I.; Lantz, T.C.; Schmitt, C. UAV photogrammetry for mapping vegetation in the low-Arctic. Arct. Sci. 2016, 2, 79-102.

28. Gevaert, C.M.; Persello, C.; Sliuzas, R.; Vosselman, G. Informal settlement classification using point-cloud and image-based features from UAV data. ISPRS J. Photogramm. Remote Sens. 2017, 125, 225-236.

29. Suomalainen, J.; Anders, N.; Iqbal, S.; Roerink, G.; Franke, J.; Wenting, P.; Hünniger, D.; Bartholomeus, H.; Becker, R.; Kooistra, L. A Lightweight Hyperspectral Mapping System and Photogrammetric Processing Chain for Unmanned Aerial Vehicles. Remote Sens. 2014, 6, 11013-11030.

30. Xie, Y.; Sha, Z.; Yu, M. Remote sensing imagery in vegetation mapping: A review. J. Plant Ecol. 2008, 1, 9-23, doi:10.1093/jpe/rtm005.

31. Laliberte, A.S.; Winters, C.; Rango, A. UAS remote sensing missions for rangeland applications. Geocarto Int. 2011, 26, 141-156.

32. Puliti, S.; Orka, O.H.; Gobakken, T.; Næsset, E. Inventory of Small Forest Areas Using an Unmanned Aerial System. Remote Sens. 2015, 7, 9632-9654.

33. Garcia-Torres, L.; Caballero-Novella, J.J.; Gómez-Candón, D.; De-Castro, A.I. Semi-Automatic Normalization of Multitemporal Remote Images Based on Vegetative Pseudo-Invariant Features. PLoS ONE 2014, 9, e91275.

34. Jha, A.R. Theory, Design, and Applications of Unmanned Aerial Vehicles; CRC Press: Boca Raton, FL, USA, 2016.

35. Watts, A.C.; Ambrosia, V.G.; Hinkley, E.A. Unmanned Aircraft Systems in Remote Sensing and Scientific Research: Classification and Considerations of Use. Remote Sens. 2012, 4, 1671-1692.

36. Aber, J.S.; Marzolff, I.; Ries, J. Small-Format Aerial Photography: Principles, Techniques and Geoscience Applications; Elsevier Science: Amsterdam, The Netherlands, 2010.

37. Gutiérrez, P.A.; López-Granados, F.; Peña-Barragán, J.M.; Jurado-Expósito, M.; Hervás-Martínez, C. Logistic regression product-unit neural networks for mapping Ridolfia segetum infestations in sunflower crop using multitemporal remote sensed data. Comput. Electron. Agric. 2008, 64, 293-306.

38. Lan, Y.; Huang, Y.; Martin, D.E.; Hoffmann, W.C. Development of an Airborne Remote Sensing System for Crop Pest Management: System Integration and Verification. Appl. Eng. Agric. 2009, 25, 607-615.

39. Fladeland, M.; Sumich, M.; Lobitz, B.; Kolyer, R.; Herlth, D.; Berthold, R.; McKinnon, D.; Monforton, L.; Brass, J.; Bland, G. The NASA SIERRA science demonstration programme and the role of small-medium unmanned aircraft for earth science investigations. Geocarto Int. 2011, 26, 157-163.

40. Primicerio, J.; Di Gennaro, S.F.; Fiorillo, E.; Genesio, L.; Lugato, E.; Matese, A.; Vaccari, F.P. A flexible unmanned aerial vehicle for precision agriculture. Precis. Agric. 2012, 13, 517-523.

41. Gonzalez-Dugo, V.; Zarco-Tejada, P.; Nicolás, E.; Nortes, P.A.; Alarcón, J.J.; Intrigliolo, D.S.; Fereres, E. Using high resolution UAV thermal imagery to assess the variability in the water status of five fruit tree species within a commercial orchard. Precis. Agric. 2013, 14, 660-678.

42. Tamminga, A.; Hugenholtz, C.; Eaton, B.; Lapointe, M. Hyperspatial Remote Sensing of Channel Reach Morphology and Hydraulic Fish Habitat Using an Unmanned Aerial Vehicle (UAV): A First Assessment in the Context of River Research and Management. River Res. Appl. 2015, 31, 379-391.

43. Turner, D.; Lucieer, A.; de Jong, M.S. Time Series Analysis of Landslide Dynamics Using an Unmanned Aerial Vehicle (UAV). Remote Sens. 2015, 7, 1736-1757. 
44. Gonçalves, J.A.; Bastos, L.; Pinho, J.L.; Granja, H. Digital Aerial Photography to Monitor Changes in Coastal Areas Based on Direct Georeferencing. In Proceedings of the 5th EARSeL Workshop on Remote Sensing of the Coastal Zone, Prague, Czech Republic, 1-3 June 2011; pp. 1-10.

45. Gonçalves, J.A.; Henriques, R. UAV Photogrammetry for Topographic Monitoring of Coastal Areas. ISPRS J. Photogramm. Remote Sens. 2015, 104, 101-111.

46. Drummond, C.D.; Harley, M.D.; Turner, I.L.; A Matheen, A.N.; Glamore, W.C. UAV Applications to Coastal Engineering. In Proceedings of the Australasian Coasts \& Ports Conference, Auckland, New Zealand, 15-18 September 2015; pp. 267-272.

47. Messinger, M.; Silman, M. Unmanned Aerial Vehicles for the Assessment and Monitoring of Environmental Contamination: An Example from Coal Ash Spills. Environ. Pollut. 2016, 218, 889-894.

48. Pereira, E.; Bencatel, R.; Correia, J.; Félix, L.; Gonçalves, G.; Morgado, J.; Sousa, J. Unmanned Air Vehicles for Coastal and Environmental Research. J. Coast. Res. 2009, II, 1557-1561.

49. Hodgson, A.; Kelly, N.; Peel, D. Unmanned Aerial Vehicles (UAVs) for Surveying Marine Fauna: A Dugong Case Study. PLoS ONE 2013, 8, e79556.

50. Rhee, D.S.; Kim, Y.D.; Kang, B.; Kim, D. Applications of Unmanned Aerial Vehicles in Fluvial Remote Sensing: An Overview of Recent Achievements. KSCE J. Civ. Eng. 2017, 1-15, doi:10.1007/s12205-017-1862-5.

51. Abdullahi, H.S.; Mahieddine, F.; Sheriff, R.E. Technology Impact on Agricultural Productivity: A Review of Precision Agriculture Using Unmanned Aerial Vehicles. In Proceedings of the Wireless and Satellite Systems: 7th International Conference on WiSATS, Bradford, UK, 6-7 July 2015; Pillai, P., Hu, Y.F., Otung, I., Giambene, G., Eds.; Revised Selected Papers; Springer International Publishing: Cham, Switzerland, 2015; pp. 388-400.

52. Felderhof, L.; Gillieson, D. Near-infrared Imagery From Unmanned Aerial Systems and Satellites Can Be Used to Specify Fertilizer Application Rates in Tree Crops. Can. J. Remote Sens. 2012, 37, 376-386.

53. Gómez-Candón, D.; De Castro, A.I.; López-Granados, F. Assessing the accuracy of mosaics from unmanned aerial vehicle (UAV) imagery for precision agriculture purposes in wheat. Precis. Agric. 2014, 15, 44-56.

54. Chu, T.; Starek, M.J.; Brewer, M.J.; Masiane, T.; Murray, S.C. UAS imaging for automated crop lodging detection: a case study over an experimental maize field. In Proceedings of the SPIE Commercial + Scientific Sensing and Imaging, Anaheim, CA, USA, 8 May 2017; Volume 10218, p. 102180E.

55. Wei, L.; Yang, B.; Jiang, J.; Cao, G.; Wu, M. Vegetation filtering algorithm for UAV-borne lidar point clouds: A case study in the middle-lower Yangtze River riparian zone. Int. J. Remote Sens. 2017, 38, 2991-3002.

56. Bendig, J.; Bolten, A.; Bareth, G. UAV-based Imaging for Multi-Temporal, very high Resolution Crop Surface Models to monitor Crop Growth VariabilityMonitoring des Pflanzenwachstums mit Hilfe multitemporaler und hoch auflösender Oberflächenmodelle von Getreidebeständen auf Basis von Bildern aus UAV-Befliegungen. Photogramm. Fernerkund. Geoinf. 2013, 2013, 551-562.

57. Vega, F.A.; Ramírez, F.C.; Saiz, Ó.P.; Rosúa, F.O. Multi-temporal imaging using an unmanned aerial vehicle for monitoring a sunflower crop. Biosyst. Eng. 2015, 132, 19-27.

58. Castaldi, F.; Pelosi, F.; Pascucci, S.; Casa, R. Assessing the potential of images from unmanned aerial vehicles (UAV) to support herbicide patch spraying in maize. Precis. Agric. 2016, 18, 1-19.

59. Willkomm, M.; Bolten, A.; Bareth, G. Non-destructive monitoring of rice by hyperspectral in-field spectrometry and UAV-based remote sensing: Case study of field-grown rice in north Rhine-Westphalia, Germany. ISPRS Int. Arch. Photogramm. Remote Sens. Spat. Inf. Sci. 2016, XLI-B1, 1071-1077.

60. Du, M.; Noguchi, N. Monitoring of Wheat Growth Status and Mapping of Wheat Yield's within-Field Spatial Variations Using Color Images Acquired from UAV-camera System. Remote Sens. 2017, 9, 289.

61. Holman, F.H.; Riche, A.B.; Michalski, A.; Castle, M.; Wooster, M.J.; Hawkesford, M.J. High throughput field phenotyping of wheat plant height and growth rate in field plot trials using UAV based remote sensing. Remote Sens. 2016, 8, 1031.

62. Ballesteros, R.; Ortega, J.F.; Hernández, D.; Moreno, M.Á. Characterization of Vitis vinifera L. canopy using unmanned aerial vehicle-based remote sensing and photogrammetry techniques. Am. J. Enol. Vitic. 2015, 21, doi:10.5344/ajev.2014.14070.

63. Long, N.; Millescamps, B.; Guillot, B.; Pouget, F.; Bertin, X. Monitoring the topography of a dynamic tidal inlet using UAV imagery. Remote Sens. 2016, 8, 387.

64. Lucieer, A.; Jong, S.M.D.; Turner, D. Mapping landslide displacements using Structure from Motion (SfM) and image correlation of multi-temporal UAV photography. Prog. Phys. Geogr. 2014, 38, 97-116. 
65. Guerra-Hernández, J.; González-Ferreiro, E.; Monleón, V.J.; Faias, S.P.; Tomé, M.; Díaz-Varela, R.A. Use of Multi-Temporal UAV-Derived Imagery for Estimating Individual Tree Growth in Pinus pinea Stands. Forests 2017, 8, 300 .

66. Wang, J.; Ge, Y.; Heuvelink, G.B.M.; Zhou, C.; Brus, D. Effect of the sampling design of ground control points on the geometric correction of remotely sensed imagery. Int. J. Appl. Earth Obs. Geoinf. 2012, 18, 91-100.

67. Fryskowska, A.; Kedzierski, M.; Grochala, A.; Braula, A. Calibration of low cost RGB and NIR UAV cameras. In Proceedings of the the International Archives of the Photogrammetry, Remote Sensing and Spatial Information Sciences, Prague, Czech Republic, 12-19 July 2016; Volume XLI-B1, pp. 817-821.

68. James, M.R.; Robson, S. Mitigating Systematic Error in Topographic Models Derived from UAV and Ground-based Image Networks. Earth Surf. Process. Landf. 2014, 39, 1413-1420.

69. Martha, T.R.; Kerle, N.; van Westen, C.J.; Jetten, V.; Vinod Kumar, K. Effect of Sun Elevation Angle on DSMs Derived from Cartosat-1 Data. Photogramm. Eng. Remote Sens. 2010, 76, 429-438.

70. Gomes-Laranjo, J.; Dinis, L.T.; Martins, L.; Portela, E.; Pinto, T.; Ara, M.C.; Díaz, I.F.; Majada, J.; Peixoto, F.; Lorenzo, S.P.; et al. Characterization of Chestnut Behavior with Photosynthetic Traits. In Applied Photosynthesis; InTech: Rijeka, Croatia, 2012.

71. Santos, C.; Zhebentyayeva, T.; Serrazina, S.; Nelson, C.D.; Costa, R. Development and characterization of EST-SSR markers for mapping reaction to Phytophthora cinnamomi in Castanea spp. Sci. Hortic. 2015, 194, 181-187.

72. Robin, C.; Lanz, S.; Soutrenon, A.; Rigling, D. Dominance of natural over released biological control agents of the chestnut blight fungus Cryphonectria parasitica in south-eastern France is associated with fitness-related traits. Biol. Control 2010, 53, 55-61.

73. Ambrosini, I.; Gherardi, L.; Viti, M.L.; Maresi, G.; Turchetti, T. Monitoring Diseases of Chestnut Stands by Small Format Aerial Photography. Geocarto Int. 1997, 12, 41-46.

74. DRAPN. Plano de Ação Nacional Para o Controlo do Inseto Dryocosmus Kuriphilus YASUMATSU (Vespa das Galhas do Castanheiro); Direção Regional de Agricultura e Pescas do Norte: Mirandela, Portugal, 2014.

75. Sartor, C.; Dini, F.; Marinoni, D.T.; Mellano, M.; Beccaro, G.; Alma, A.; Quacchia, A.; Botta, R. Impact of the Asian wasp Dryocosmus kuriphilus (Yasumatsu) on cultivated chestnut: Yield loss and cultivar susceptibility. Sci. Hortic. 2015, 197, 454-460.

76. Mozas-Calvache, A.T.; Pérez-García, J.L.; Cardenal-Escarcena, F.J.; Mata-Castro, E.; Delgado-García, J. Method for photogrammetric surveying of archaeological sites with light aerial platforms. J. Archaeol. Sci. 2012, 39, 521-530.

77. Xiang, H.; Tian, L. Development of a low-cost agricultural remote sensing system based on an autonomous unmanned aerial vehicle (UAV). Biosyst. Eng. 2011, 108, 174-190.

78. Laliberte, A.S.; Herrick, J.E.; Rango, A.; Winters, C. Acquisition, Orthorectification, and Object-based Classification of Unmanned Aerial Vehicle (UAV) Imagery for Rangeland Monitoring. Photogramm. Eng. Remote Sens. 2010, 76, 661-672.

79. ICNF. Relatório Final IFN5-FloreStat; ICNF: Lisbon, Portugal, 2010.

80. Soares, A. Geoestatística Para as Ciências da Terra e do Ambiente; IST-Instituto Superior Técnico: Lisbon, Portugal, 2000.

81. Sousa, A.; Muge, F. Elementos de Geoestatística; IST-Instituto Superior Técnico: Lisbon, Portugal, 1990.

82. Dong, X.; Zhou, Y.; Ren, Z.; Zhong, Y. Time-varying Formation Control for Unmanned Aerial Vehicles with Switching Interaction Topologies. Control Eng. Pract. 2016, 46, 26-36.

83. Bounous, G.; Conedera, M. Il Castagno: Risorsa Multifunzionale in Italia e nel Mondo, 1st ed.; Edagricole: Bologna, Italy, 2014; from 2002, now updated and enlarged.

84. Martins, L.; Castro, J.; Marques, C.; Abreu, C. Assessment of the spread of chestnut ink disease from 1995 to 2005 using aerial photography and geostatistical methods. Int. Chestnut Symp. 2009, 844, 349-354.

85. Gehring, E.; Pezzatti, G.B.; Krebs, P.; Mazzoleni, S.; Conedera, M. On the applicability of the pipe model theory on the chestnut tree (Castanea sativa Mill.). Trees 2015, 29, 321-332.

86. Martins, L.M.; Lufinha, M.I.; Marques, C.P.; Abreu, C.G. Small format aerial photography to assess chestnut ink disease. For. Snow Landsc. Res. 2001, 73, 357-360.

87. Teodoro, A.; Taveira-Pinto, F.; Santos, I. Morphological and statistical analysis of the impact of breakwaters under construction on a sand spit area (Douro River estuary). J. Coast. Conserv. 2014, 18, 177-191. 
88. Bio, A.; Bastos, L.; Granja, H.; Pinho, L.; Gonçalves, J.; Henriques, R.; Rodrigues, D. Methods for Coastal Monitoring and Erosion Risk Assessment: Two Portuguese Case Studies. J. Integr. Coast. Zone Manag. 2015, 15, 47-63.

89. Dandois, P.J.; Olano, M.; Ellis, C.E. Optimal Altitude, Overlap, and Weather Conditions for Computer Vision UAV Estimates of Forest Structure. Remote Sens. 2015, 7, 13895-13920.

90. Troen, I.; Lundtang Petersen, E. European Wind Atlas; Riso National Laboratory: Roskilde, Denmark, 1989.

91. Rehak, M.; Skaloud, J. Fixed-wing micro aerial vehicle for accurate corridor mapping. ISPRS Ann. Photogramm. Remote Sens. Spat. Inf. Sci. 2015, II-1/W1, 23-31.

(C) 2017 by the authors. Licensee MDPI, Basel, Switzerland. This article is an open access article distributed under the terms and conditions of the Creative Commons Attribution (CC BY) license (http://creativecommons.org/licenses/by/4.0/). 